\title{
RELATIONSHIP BETWEEN FLASH POINTS OF SOME BINARY BASE OILS AND THEIR CONSTITUENT OILS
}

\author{
Mahboube Shirani ${ }^{1}$, Abolfazl Semnani ${ }^{1 *}$ Hamid Shakoori Langeroodi $^{2}$ and Hedayat Haddadi $^{1}$ \\ ${ }^{1}$ Department of Chemistry, Faculty of Sciences, University of Shahrekord, Shahrekord, Iran \\ ${ }^{2}$ Barzin Sepand Sepahan Company, Dehagh Industrial City, Isfahan, Iran
}

(Received July 17, 2013; revised December 12, 2014)

\begin{abstract}
Miscellaneous binary blends containing solvent neutral-150 (SN-150), solvent neutral-500 (SN500), Bright Stock-150 (BS-150), polyalphaolephin-6 (PAO-6), PAO-100, a processed low sulfur waxy material, and polyisobutene-600 (PIB-600) were prepared and their corresponding flash point (FP) were measured. By using the measured FP data, the plots of FP vs. weight percentage of minor component were drawn and the equations due to each plot were derived. Except PIB-600 containing mixtures, linear relation between FP of blends and weight percentage of constituents has been observed. Also the obtained data indicate that except PIB600 containing blends the FP of such mixtures can be calculated from the summation of "FP $\times$ weight ratio" of each component. In the case of PIB-600 containing blends, nonlinear and decreasing, nonlinear and increasing were observed as well as constant trends of FP vs. weight percentage of minor component. The observations have been interpreted based on the composition, molecular weight, and interactions of the constituents of the base oils.
\end{abstract}

KEY WORDS: Flash point, Binary lubricants, Tribotests, Mineral oils, Synthetic oils, Semisynthetic oils

\section{INTRODUCTION}

One of the earliest known identifiers of oil's physical properties is its flash point. References to the test, associated with lamp oils, date back to the mid-19th century. Different instruments, procedures, and applications of flash point testing have been described in the literature [1]. Like viscosity, the flash point test has always been a standard part of a lubricant's specification. According to ASTM (American Society for Testing and Materials), which first standardized the test in 1924, the flash point is the lowest temperature at which an ignition source causes the vapors of the specimen (lubricant) to ignite under specified conditions [2].

Originally, the flash point was developed for the purpose of determining the fire hazard of fuels and oils being stored or transported. However, combined with other tests such as viscosity, viscosity index, and specific gravity, the flash point can help to reveal both the quality of the crude oil from which the lubricant was derived and the quality of the refining process. The flash point can also identify whether the base oil was a wide or narrow single cut or whether it represents a blend of two fractions (two base oils of different viscosities mixed together). And, the flash point may give some indication about the volatility and content of the most volatile components of the test oil, base oil cracking, contamination, wrong oil/mixed oil, subtractions from the oil $[1,3,4]$.

Although the experimental measurement of flash point is not complicated, the extension of theoretical methods for its prediction in blends will help in the saving of cost and time. In addition, an initial knowledge about the flash point of mixtures can be regarded as an effective tool in designing the target blends. Several studies have been performed to find the relationship between structure and flash points of organic compounds [5-12] and fuels [13, 14]. However, to the best of our knowledge, there is little investigation in the case of blends of lubricating oil [15, $16]$.

We have recently been involved in the investigation of lubricating oils [17-22]. In this paper we have performed an investigation on the relationship between flash points of some binary

*Corresponding author. E-mail: a.semnani1341@gmail.com 
base oils and weight ratios of their components. Because of extensive usage of SN-150, SN-500, and BS-150 in the formulation of engine oils, the recent mineral oils were chosen for study. On the other hand, the good miscibility of PAO-6, PAO-100 and PIB-600 with selected mineral oils as well as their availability was the main reason for selection of these synthetic oils. It should be mentioned that all the mixture applied in this study are misible.

\section{EXPERIMENTAL}

All of the flash points were measured according to ASTM D-92 procedure by flash point tester Herzog Model HC 852. The group (III) base oil was prepared according to the following procedure: $100 \mathrm{~kg}$ of low sulfur waxy material from Isomax unit was transferred to a batch kettle. Then, $10 \mathrm{~kg}$ of urea and $1 \mathrm{~kg}$ of methanol were added to it, and the resulting material was mixed for 8 hours. A white product was obtained. Passing the recent mixture from the filter press caused the separation of oil from the wax. The oil was transferred to another kettle and $\mathrm{H}_{2} \mathrm{SO}_{4} 98 \%$ (10\% of the weight of the oil) was added to it and the resulting mixture was mixed for eight hours. Afterwards, the mixing was stopped and allowed to settle the precipitates. The oil was transferred to another kettle and mixed with zeolite (30\% of the weight of the oil) for one hour. Finally, the discolored oil was separated from zeolite by filter press. The purified oil was used for the next steps.

\section{RESULTS AND DISCUSSION}

The selected base oils are introduced in Table 1 . The category of oils as well as flash point values of each of them is also given. As it can be seen both mineral and synthetic oils have been chosen. In addition, the data indicate that the flash point values due to mineral and synthetic oils differ significantly. Consequently, it is expected that through stepwise alteration of weight ratios in binary mixtures, the gaps between flash points would be high enough. So the flash point monitoring will be facilitated and the interpretation of the resulting data will be reliable.

Table 1. Flash point of selected oils.

\begin{tabular}{|c|c|c|c|}
\hline Base oil & Category & Company & Flash point $\left({ }^{\circ} \mathrm{C}\right)$ \\
\hline SN-150 & Mineral & Iranol & 210 \\
\hline SN-500 & Mineral & Iranol & 245 \\
\hline BS-150 & Mineral & Iranol & 270 \\
\hline PAO-6 & Synthetic & Exxon Mobil & 245 \\
\hline PAO-100 & Synthetic & Exxon Mobil & 290 \\
\hline PIB-600 & Synthetic & ELA & 250 \\
\hline Group (III) & Mineral & Prepared & 200 \\
\hline
\end{tabular}

Blends of SN-150: SN-500

The plots of flash point of different binary blends of SN-150: SN-500 (full mineral mixtures) vs. weight percentage of SN-150 is shown in Figure 1. The weight percentages of SN-150 vary from 5 to 50 . As it can be seen, (i) upon addition of SN-150, the flash point decreased systematically and (ii) the variations of flash point vs. weight percentage is linear and obeys the following equation:

$(\mathrm{FP})_{\text {blend }}=-0.349(\mathrm{wt} \%)_{\mathrm{SN}-150}+244.6$

$\left[(\mathrm{FP})_{\mathrm{SN}-150} \times(\text { weight ratio })_{\mathrm{SN}-150}\right]+\left[(\mathrm{FP})_{\mathrm{SN}-500} \times(\text { weight ratio })_{\mathrm{SN}-500}\right]$ 
(a)

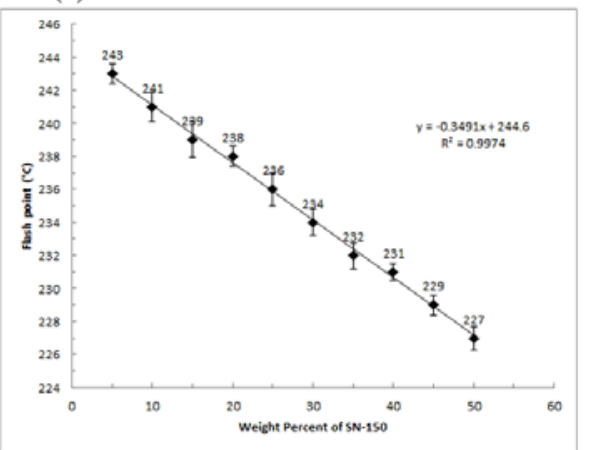

(c)

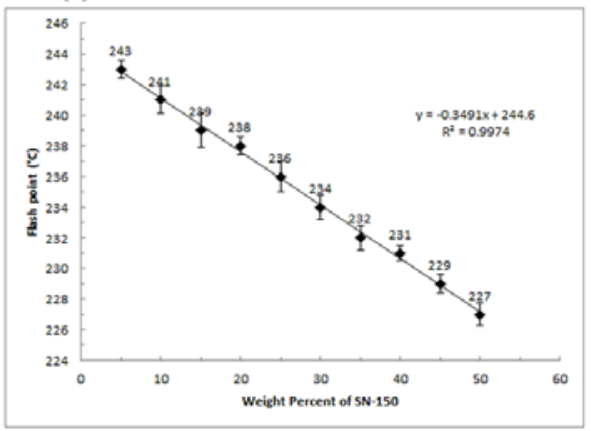

(b)

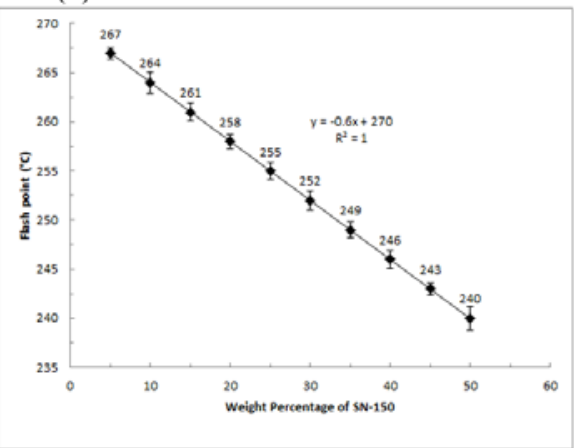

Figure 1. (a) Plot of flash point of different SN-500: SN-150 blends vs. weight percentage of SN-150, (b) Plot of flash point of different BS-150: SN-150 blends vs. weight percentage of SN-150, (c) Plot of flash point of different PAO-6: SN-150 blends vs. weight percentage of $\mathrm{SN}-150$.

As it is observed, there is an excellent agreement between calculated and obtained data. So, by using the above relation a blend with predetermined flash point can easily be designed. The obtained results can be simply explained as follow:

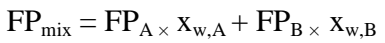

where $\mathrm{FP}_{\text {mix }}, \mathrm{FP}_{\mathrm{A}}, \mathrm{FP}_{\mathrm{B}}$ are the flash points of the mixture, the component $\mathrm{A}$ and component $\mathrm{B}$ respectively; and $\mathrm{X}_{\mathrm{w}, \mathrm{A}}, \mathrm{x}_{\mathrm{w}, \mathrm{B}}$ are the mass proportion (or mass fraction) of $\mathrm{A}$ and $\mathrm{B}$. But $\mathrm{x}_{\mathrm{w}, \mathrm{A}}+\mathrm{x}_{\mathrm{w}, \mathrm{B}}$ $=1$ and then $\mathrm{x}_{\mathrm{w}}, \mathrm{B}=1-\mathrm{x}_{\mathrm{w}, \mathrm{A}}$ substitute in the previous expression to the following equation:

$\mathrm{FP}_{\text {mix }}=\left(\mathrm{FP}_{\mathrm{A}}-\mathrm{FP}_{\mathrm{B}}\right) \times \mathrm{X}_{\mathrm{w}, \mathrm{A}}+\mathrm{FP}_{\mathrm{B}}$

That is the other form of the equation (3), but it is just the expression of a straight line with slope $\left(\mathrm{FP}_{\mathrm{A}}-\mathrm{FP}_{\mathrm{B}}\right)$ and zero interception $\mathrm{FP}_{\mathrm{B}}$. Equation (4) can be written in another form as equation (5):

$\mathrm{FP}_{\text {mix }}=\mathrm{a} \times \mathrm{x}_{\mathrm{w}, \mathrm{A}}+\mathrm{b}$

Since it is a linear relationship, the constant of ' $a$ ' should be close to $\mathrm{FP}_{\mathrm{A}}-\mathrm{FP}_{\mathrm{B}}$ and the constant 'b' close to $\mathrm{FP}_{\mathrm{B}}$. 
Blends of SN-150: BS-150

The corresponding plot of flash point vs. wt\% of SN-150 is shown in Figure 1 (b). Similar to SN-150: SN-500 mixtures (Figure 1 (a)), decreasing as well as linear trend is observed. It is interesting to note that the absolute slope of the SN-150: BS-150 (Figure 1 (b)) is nearly twice greater than the slope of SN-150: SN-500 plot (Figure 1 (a)). This means that relative to SN500 , the addition of SN-150 to BS-150 does have greater effect on flash point of the resulting blend. Upon analysis of the data due to Figure 1 (b) it can be shown that the flash point of every SN-150: BS-150 blend can be predicted from the following relation:

$\left[(\mathrm{FP})_{\mathrm{SN}-150} \times(\text { weight ratio })_{\mathrm{SN}-150}\right]+\left[(\mathrm{FP})_{\mathrm{BS}-150} \times(\text { weight ratio })_{\mathrm{BS}-150}\right]$

The above relation is similar to equation (2) which was derived for SN-150: SN-500 mixtures.

Blends of PAO-6 with SN-150, BS-150, and PAO-100

The plots of flash points of binary blends of SN-150: PAO-6, BS-150: PAO-6 and PAO-6: PAO-100 are shown in Figures 1 (c) and Figure 2. In all cases linear relationships are observed. The corresponding results are given in Table 2.

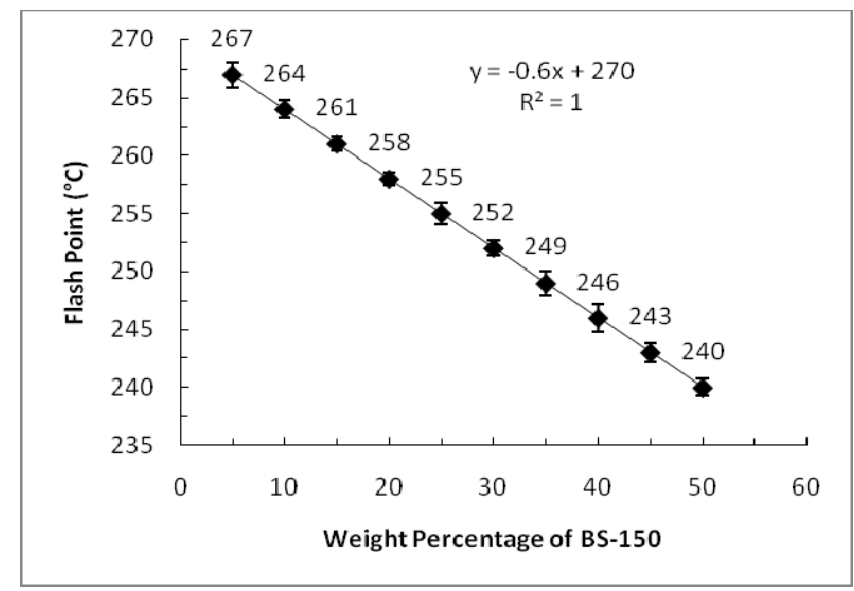

Figure 2. Plot of flash point of different PAO-6: Bright Stock-150 blends vs. Weight percentage of Bright Stock-150

Table 2. The slope and intercept obtained from the plots due to flash points of binary blends of SN-150: SN-500, SN-150: PAO-6, BS-150: PAO-6, and PAO-6: PAO-100.

\begin{tabular}{|c|c|c|c|c|}
\hline Mixture (A:B) & $\mathrm{a} / \mathrm{K}$ & $\mathrm{b} / \mathrm{K}$ & $\left(\mathrm{FP}_{\mathrm{A}}-\mathrm{FP}_{\mathrm{B}}\right)$ & $\mathrm{FP}_{\mathrm{B}}$ \\
\hline SN-150: SN-500 & -34.9 & 244.6 & -35 & 245 \\
\hline SN-150:BS-150 & -0.6 & 270 & -0.6 & 270 \\
\hline SN-150:PAO-6 & -34.9 & 244.6 & -35 & 245 \\
\hline BS-150:PAO-6 & -0.6 & 270 & -0.6 & 270 \\
\hline PAO-6: PAO-100 & -0.452 & 289.9 & 0.5 & 290 \\
\hline
\end{tabular}

Also, similar to previous cases, the FP values can be obtained from the following relations: 


$$
\begin{aligned}
& (\mathrm{FP})_{\text {blend }}=\left[(\mathrm{FP})_{\mathrm{SN}-150} \times(\text { weight ratio })_{\mathrm{SN}-150}\right]+\left[(\mathrm{FP})_{\mathrm{PAO}-6} \times(\text { weight ratio })_{\mathrm{PAO}-6}\right] \\
& (\mathrm{FP})_{\text {blend }}=\left[(\mathrm{FP})_{\mathrm{PAO}-6} \times(\text { weight ratio })_{\mathrm{PAO}-6}\right]+\left[(\mathrm{FP})_{\mathrm{BS}-150} \times(\text { weight ratio })_{\mathrm{BS}-150}\right] \\
& (\mathrm{FP})_{\text {blend }}=\left[(\mathrm{FP})_{\mathrm{PAO}-6} \times(\text { weight ratio })_{\mathrm{PAO}-6}\right]+\left[(\mathrm{FP})_{\mathrm{PAO}-100} \times(\text { weight ratio })_{\mathrm{PAO}-100}\right]
\end{aligned}
$$

\section{Blends of PIB-600: SN-500}

The plot due to flash points of different binary blends of PIB-600: SN-500 vs. weight percentage of PIB is shown in Figure 3. As it is observed upon stepwise increase of PIB-600, FP will increase until 35\%, and will then stopped, i.e. in contrast to previous cases a linear relationship is not observed.

It is interesting to note that PIB-600 and SN-500 do have nearly the same FP values (Table 1). So, it is expected that the FP of their blends to be close to the corresponding value due to individual oils. However, this is not occurred and the blending does have increasing effect on the property, i.e. the FP of blends are more than the FP of each of the oils.

\section{Blends of PIB: PAO-6}

In contrast to PIB-600:SN-500 blends, the blends of PIB-600:PAO-6 (again two base oils with nearly equal flash point) are lower than their components (Figure 5) and upon addition of PIB600 to PAO-6, decreasing trend is observed.

\section{Blends of PIB-600: Group (III)}

Addition of PIB to Group (III) does not alter the flash point (Table 1) and despite the FP of PIB is more than Group (III), increasing effect is not observed. This is a very interesting feature, and may be applied for the development of new class of additives which can buffer the FP.

The results show that there is what is called a "deviation of a property" in this case the "deviation of Flash Point" that is defined as the difference of the experimental property and the linear relationship. They usually indicate some kind of interaction between the components. It should be mentioned that for mixture with linear relationships which explained previously the deviation of FP is zero. For mixtures like PIB: PAO-6 and PIB-600: Group (III) with non-linear relationships the deviation from linearity is very large. The FP of PIB-600, SN-500 and PAO-6 are very similar, however, mixtures of 50\% change the FP around $20 \mathrm{~K}$. There is a whole treatment for this deviations and even an empirical equation (Redlich-Kister) [23].

\section{General discussion}

Different equations have been developed for the prediction of FP of pure substances [23]. Through versatile equations FP has been related to boiling point [6, 24-26], atomic composition [28], and standard energy of vaporization [27]. Quantitative Structure Property Relationship (QSPR) method [7, 28] has also been employed for the estimation of FP. In addition, equations have been proposed for the estimation of binary [31], ternary [29], and multi component mixtures [23, 27].

Development of recent methods to lubricants involves detailed compositional information. Since this is a difficult task, a vacancy of data in this field is observed $[15,16]$. The problem is more tedious in the case of mineral oils which is relative to other base oils and have more complex compositions.

Beside the ignition and through gradual heating of multi component base oil, the concentration of different constituents in the vapor phase will be increased. However, the first flash will be produced by the momentary ignition of the constituent, which its concentration in the vapor phase has been reached to critical value, i.e. a compound with lowest flammability limit [30], which is abbreviated as LFL. 
For an ideal solution of lubricant in which the concentrations of all constituents are equal, it is expected that the flash point of the mixture to be determined by the constituent with lowest LFL. The violation of the solution behavior from the Raoult law [31], as well as inequality of the concentrations of blend components, will change the situation. This may cause that the flash point to be determined by other components.

Comparison of the aromatic, naphthenic, and paraffinic components of different employed mineral oils (Table 3) indicate that SN-150 does have the highest naphthenic content. On the other hand it is well known that the FP of recent contents is less than aromatic and paraffinic ones [32]. So, the lowest FP of SN-150 relative to SN-500 or BS-150 is not unexpected (Table 1). In addition because of higher naphthenic components of SN-150 containing blends the lower flash point of such combinations (Figure 1) is not unusual. Also the linear correlation between FP and weight percentage of SN-150 in different SN-150 containing blends (Figure 1) can be attributed to the linear relation between weight percentage of SN-150 and weight percentage of naphthenic In fact such a relation indicate that one or several of the compounds which exist in naphthenic component play the main role in producing the flash point and the recent compound show ideal behaviour, i.e. does not have special interactions with other existing constituents. Similar interpretation can be applied for the linear relation between FP and weight percentage of BS-150 in different PAO-6: BS-150 mixtures (Figure 2).

Despite naphthenic content in BS-150 is less than SN-150 (Table 3) its absolute quantity is considerable. So observation of linear relation between FP and weight percentage of BS-150 is not unusual. The average molecular weight of PAO-6 is less than PAO-100. So, the volatility of PAO-6 is more than PAO-100. This means that in a mixture of PAO-6 and PAO-100 the FP will mainly be determined by PAO-6 constituents. The linear relation between FP and weight percentage of PAO-6 in PAO-6: PAO-100 blends (Figure 3) indicate that PAO-6 and PAO-100 constituents form nearly ideal solutions.

Table 3. The percentages of paraffinic, naphthenic and aromatic content in different mineral base oils.

\begin{tabular}{|c|c|c|c|}
\hline Base oil & Paraffinic \% & Naphthenic \% & Aromatic \% \\
\hline SN-150 & 66.9 & 29.7 & 3.4 \\
\hline SN-500 & 67.3 & 28.5 & 4.2 \\
\hline BS-150 & 68.4 & 25.4 & 6.4 \\
\hline
\end{tabular}

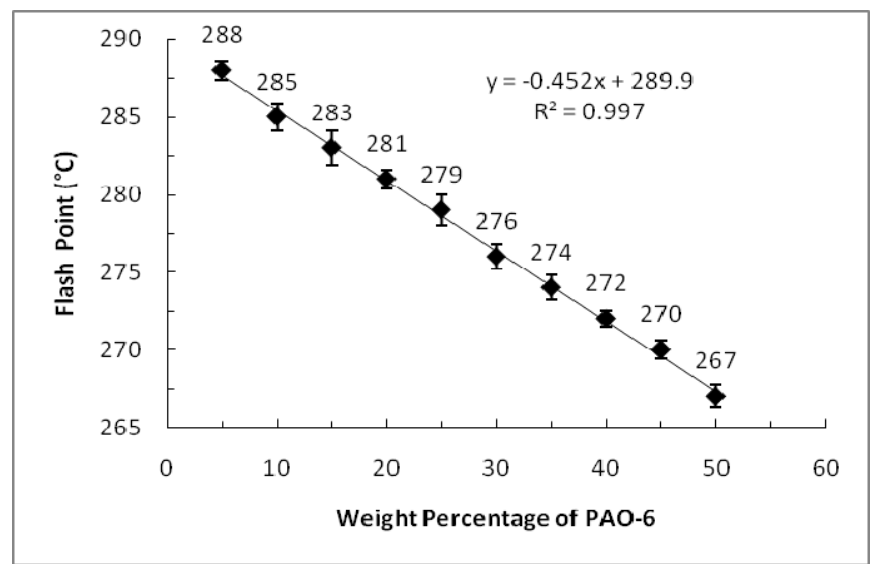

Figure 3. Plot of flash point of different PAO-100: PAO-6 blends vs. Weight percentage of PAO-6. 
Finally, the interesting behavior of PIB containing blend (Figures 4-6) can be assigned to non-ideal behavior of such blends. This probably originates from strong interaction of constituents of the blends. Moreover, the flash point behaviors for miscible mixtures and immiscible mixtures are quite different. The mixtures displayed in Figures 1 to 3 seemed to be miscible mixtures, the ones displayed in Figures 4 and 5 seemed to be partially miscible mixtures, and the ones displayed in Figure 6 seemed to be almost completely immiscible mixture [33-39].

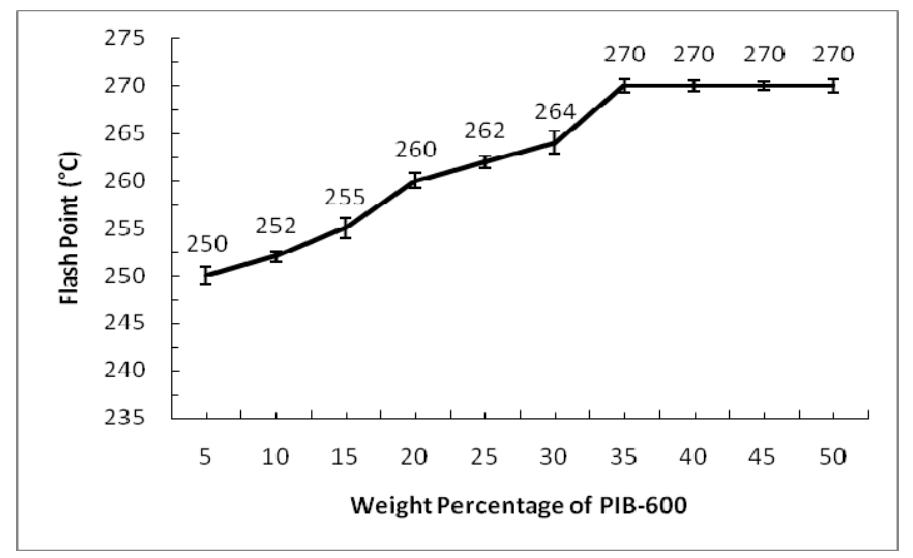

Figure 4. Plot of flash point of different PIB- 600: SN-500 blends vs. Weight percentage of PIB600.

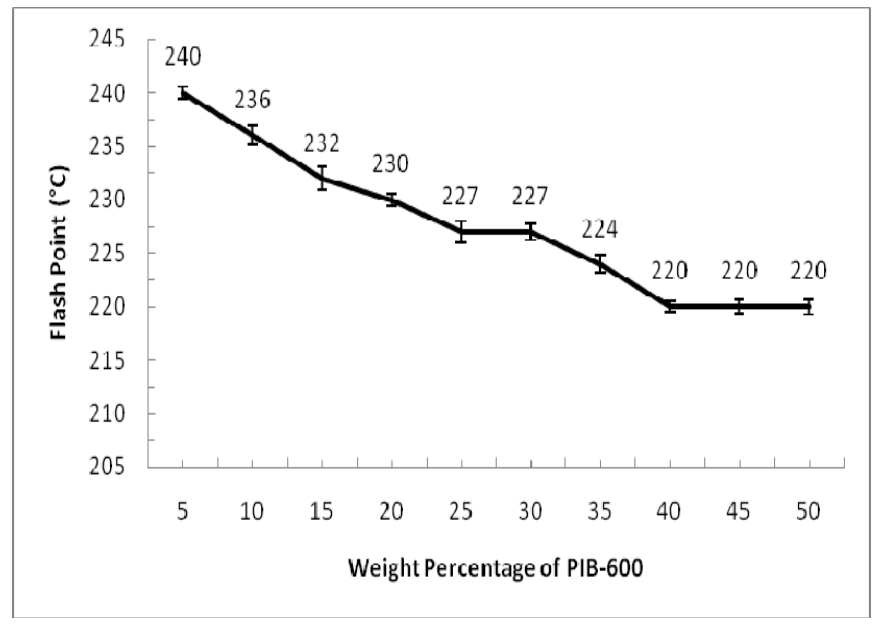

Figure 5. Plot of flash point of different PIB-600: PAO-6 blends vs. Weight percentage of PIB600 . 


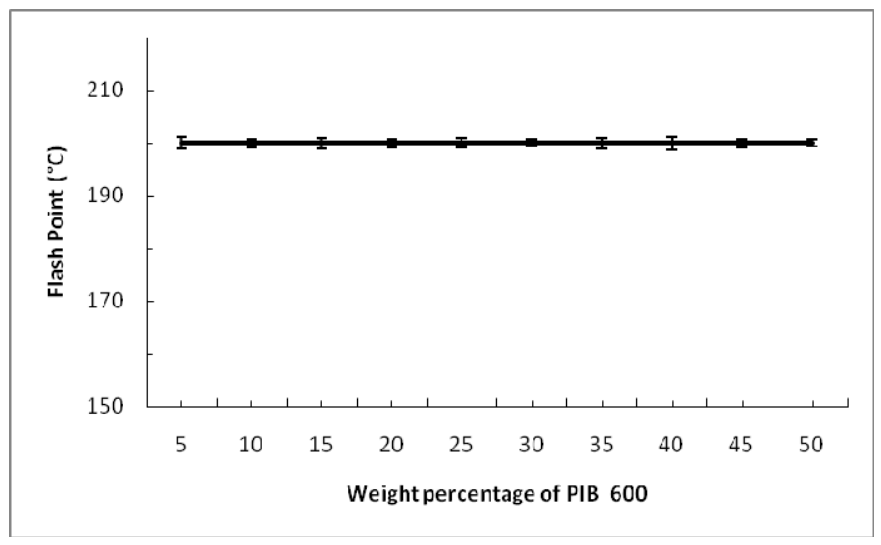

Figure 6. Plot of flash point of different PIB-600: Group (III) blends vs. Weight percentage of PIB-600.

\section{CONCLUSIONS}

Based on the obtained results it can be concluded that: (i) A linear relation exists between the FP of different investigated blends and weight percentage of their minor component. The blends which contain PIB-600 do not show such behavior. (ii) The FP of binary base oils in which the FP of constituents are not equal, will be lower than the FP of the content with greater FP. In the case of PIB-600 containing blends such behavior is not observed. (iii) The flash points of binary base oil mixtures can be predicted from the following equation: $\mathrm{FP}_{\text {blend }}=\left[(\mathrm{FP})_{1} \times\right.$ (weight ratio $\left.\left.)_{1}\right]+\left[(\mathrm{FP})_{2} \times \text { (weight ratio }\right)_{2}\right]$. The blends of PIB-600 do not obey the above equation. (iv) Despite FP values of PIB-600 and SN-500 are nearly equal. The FP of their mixtures is lower than the corresponding value of each component. (v) Despite the flash points of PIB-600 and PAO-6 are nearly equal. The FP of their mixtures is greater than the corresponding value of each component, despite the flash point of PIB is greater than Group (III). Upon stepwise addition of the former to the later, mixtures with constant FP values will be obtained. This interesting feature may be applied for the development of new class of additives which can buffer the FP.

\section{ACKNOWLEDGEMENTS}

The financial support of this work by research council of University of Shahrekord is highly appreciated. The authors were also partially supported by the Center of Excellence for Mathematics, University of Shahrekord.

\section{REFERENCES}

1. Fitch, J.C.; Troyer, D.D. Oil Analysis Basics, Noria Corporation, 1999.

2. Wray, H.A. Manual on Flash Point Standards and Their Use, ASTM: West Conshohoken, Pennisilia; 1992.

3. Pirro, T.M.; Wessol, A.A. (Eds.) Lubrication Fundamentals, Marcel Dekker: New York; 2001.

4. Hunt, T.; Evans, J. Oil Analysis Handbook, Coxmoor Publishing Company: Oxford; 2004.

5. Suzuki, T.; Ohtaguchi, K.; Koide, K. J. Chem. Eng. Jpn. 1991, 24, 258.

6. Satyanarayana, K.; Rao, P.G. J. Hazard. Mater. 1993, 32, 81.

7. Tetteh, J.; Suzuki, T.; Metcalfe, E.; Howells, S. J. Chem. Inf. S. Comput. Sci. 1999, 39, 491. 
8. Carroll, F.A.; Lin, C.-Y.; Quina, F.H. Energy and Fuels 2010, 24, 392.

9. Carroll, F.A.; Lin, C.-Y.; Quina, F.H. Ind. Eng. Chem. Res. 2011, 50, 4796.

10. Carrol, F.A.; Lin, C.-Y.; Quina, F.H. Energy and Fuels 2010, 24, 4854.

11. Gharagheizi, F.; Eslamimanesh, A.; Mohammadi, A.H.; Richon, D. Ind. Eng. Chem. Res. 2011, 50, 5877.

12. Vazhev, V.V.; Aldabergenov, M.K.; Vazheva, N.V. Petrol. Chem. 2006, 46, 136.

13. Yuan, H.; Yang, B.; Yang, J. J. Am. Oil. Chem. Soc. 2009, 86, 375.

14. Demirbas, A. Fuel 2008, 87, 1743.

15. Thiele, E.W. Ind. Eng. Chem. 1927, 19, 259.

16. Costa, D.P. Lubrication Eng. 1967, 23, 395.

17. Langeroodi, H.S.; Semnani, A. Afr. J. Pure Appl. Chem. 2009, 3, 241.

18. Semnani, A.; Langeroodi, H.S. Petrol. Sci. Technol. 2013, 31, 2089.

19. Langeroodi, H.S.; Semnani, A. Petrol. Sci. Technol. 2013, 31, 2219.

20. Ashrafi, J.; Semnani, A.; Langeroodi, H. S. Petrol. Sci. Technol. 2013, 31, 2056.

21. Rahimi, B.; Semnani, A.; Nezamzadeh-Ejhieh, A.; Langeroodi, H.S.; Davood, M.H. J. Anal. Methods Chem. 2012, 2012, 1.

22. Rahimi, B.; Semnani, A.; Nezamzadeh-Ejhieh, A.; Langeroodi, H.S.; Davood, M.H. Global J. Front. Res. 2012, 12, 9.

23. Hirostova, M.; Tchaoushev, S. J Univ. Chem. Technol. Metallurgy 2006, 41, 291.

24. Bodhurta, F.P. Industrial Explosion Prevention and Protection, McGraw-Hill: New York; 1980.

25. Hshieh, F. Fire Mat. 1997, 21, 277.

26. Prugh, R.W. J. Chem. Educ. 1973, 50, $2,85$.

27. Catoire, L.; Paulmier, S. J. Phys. Chem. Ref. Data 2006, 35, 9.

28. Patel, S.J.; Ng, D.; Mannan, M.S. Ind. Eng. Chem. Res. 2009, 48, 7378.

29. Gmehling, J.; Rasmussen, P. Eng. Chem. Fund. 1982, 21, 186.

30. Hustad, J.E. Combust. Flame 1988, 71, 283.

31. Atkins, P.; Paula, J. Physical Chemistry, Oxford University Press: Oxford; 2006.

32. Hobson, G.D.; Pohl, W. Modern Petroleum Technology, John Wiley and Sons: New York; 1973.

33. Liaw, H.J.; Gerbaud, V.; Li, Y.H. Fluid Phase Equilibr. 2011, 300, 70.

34. Liaw, H.J.; Gerbaud, V.; Chiu, C.Y. J. Chem. Eng. Data 2010, 55, 134.

35. Liaw, H.J.; Gerbaud, V.; Chen, C.C.; Shu, C.M. J. Hazard. Mater. 2010, 177, 1093.

36. Liaw, H.J.; Lu, W.H.; Gerbaud, V.; Chen, C.C. J. Hazard. Mater. 2008, 153, 1165.

37. Liaw, H.J.; Chiu, Y.Y. J. Hazard. Mater. 2006, 137, 38.

38. Liaw, H.J.; Tang, C.L.; Lai, J.S. Combust. Flame 2004, 138, 308.

39. Liaw, H.J.; Lee, Y.H.; Tang, C.L.; Hsu, H.H.; Liu, J.H. J. Loss Prevent. Proc. 2002, 15, 429. 